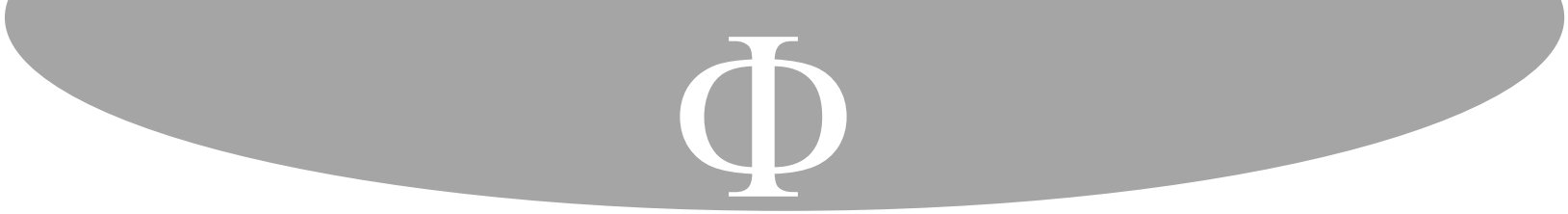

\title{
La destinación del hombre tras el repliegue trascendente de Dios en el Freiheitsschrift (1809) de Schelling (1775-1854)*
}

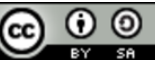

Juan José Rodríguez**

Universidad de Buenos Aires

Buenos Aires, Argentina

Para citar este artículo: Rodríguez, Juan José. «La destinación del hombre tras el repliegue trascendente de Dios en el Freiheitsschrift (1809) de Schelling (1775-1854)». Franciscanum 176, Vol. 63 (2021): 1-26.

\section{Resumen}

En este trabajo analizamos las consecuencias antropológicas del modelo de explicación metafísico y cosmológico schellinguiano que, desde 1809 en adelante, confiere al mundo y al hombre, bajo las categorías de la finitud, el mal y la libertad humana, un nuevo rol central que se desmarca de su pensamiento monista e inmanente previo (1801-1804). La hipótesis que defendemos consiste en afirmar que las críticas a las nociones de inmanencia e identidad a partir de los conceptos de separación (Zertrennung, Scheidung) y fundamento (Grund) sitúan al hombre en un rol privilegiado para continuar en la historia humana, obra de la libertad, el proceso iniciado por Dios con la creación de la naturaleza. Sin embargo, este repliegue de Dios respecto del destino del mundo, que permite al individuo humano tomar las riendas de su destinación y de asumir sobre sí toda su responsabilidad metafísica y moral, nos sitúa frente al escenario problemático de la pérdida de la universalidad y del ateísmo que vinculan a nuestro autor a las tradiciones filosóficas contemporáneas. Esta es la diferencia

\footnotetext{
* El presente trabajo se inscribe dentro de una línea de investigación doble. Desde el punto de vista individual se enmarca en la maestría Erasmus Mundus Europhilosophie (2019-2021), en particular en la investigación final sobre el concepto de libertad en Kant (1781/1790) y Schelling (1804/1811) auspiciada por las universidades de Wuppertal (Alemania) y Carolina de Praga (República Checa). Asimismo, recoge influencias de estudios actuales y pretéritos sobre la metafísica y antropología de Spinoza, financiados por la Universidad de Buenos Aires y el Instituto de Filosofía «Dr. Alejandro Korn» (2017-). Colectivamente, forma parte de los esfuerzos del proyecto UBACyT y del grupo de investigación sobre el concepto de «potencia» desde Platón hasta Schelling ambos dirigidos por el Dr. José González Ríos y la Dra. Ivana Costa (2018-).

${ }^{* *}$ Licenciado en filosofía (BA/MA) por la Universidad de Buenos Aires (UBA) con una tesis sobre el concepto de libertad en el Freiheitsschrift de Schelling, titular de la maestría Eramus Mundus Europhilosophie de la comisión europea por las universidades de Toulouse Jean Jaures (Francia), Wuppertal (Alemania) y Carolina (Praga) con una tesis sobre libertad e imaginación estéticas en Kant y Schelling, y doctorando de la UBA a partir de un proyecto que analiza la vinculación problemática entre libertad y sistema en la metafísica intermedia de Schelling. Es adscripto del Instituto de Filosofía «Dr. Alejandro Korn» de la Facultad de Filosofía y Letras de la UBA con un proyecto referido a la relación entre metafísica y antropología en Spinoza y miembro activo del grupo de investigación sobre el concepto de potencia desde Platón hasta Schelling dirigido por el Dr. José González Ríos. Ha sido titular de becas de investigación de grado con proyectos relativos a Spinoza, publicado artículos sobre filosofía moderna e idealismo en revistas y libros colectivos, así como expositor en diversos congresos nacionales e internacionales. ORCID: https://orcid.org/0000-0002-1585-3992. Contacto: juanjo_r_012@hotmail.com
} 


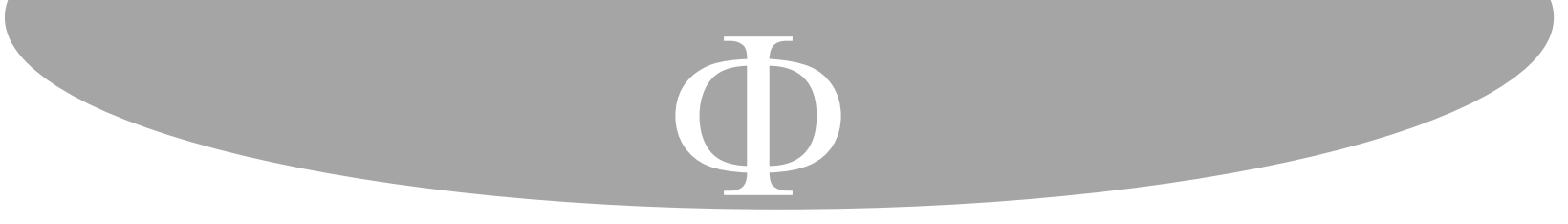

esencial, pues, entre Dios y el hombre, o como veremos, entre el Dios que es y el Dios que deviene.

Palabras clave

Schelling, hombre, Dios, finitud, inmanencia.

\title{
The destiny of man after the transcendent withdrawal of God in Schelling's Freiheitsschrift (1809) (1775-1854)
}

\begin{abstract}
In this work we analyse the anthropological consequences of the Schellinguian metaphysical and cosmological model of explanation that, from 1809 onwards, confers on the world and on man, under the categories of finitude, evil and human freedom, a new central role that is unmarked of his previous monistic and immanent thought (1801-1804). The hypothesis that we defend consists of affirming that the criticisms of the notions of immanence and identity based on the concepts of separation (Zertrennung, Scheidung) and ground (Grund) place man in a privileged role to continue in human history, the work of freedom, the process initiated by God with the creation of nature. However, this withdrawal of God with respect to the destiny of the world, which allows the human individual to take control of his destiny and to assume all his metaphysical and moral responsibility, places us in front of the problematic scenario of the loss of the universality and of atheism that link our author to contemporary philosophical traditions. This is the essential difference, then, between God and man, or as we shall see, between the God who is and the God who becomes.
\end{abstract}

\section{Keywords}

Schelling, man, God, finitude, immanence.

\section{Introducción}

En su escrito Investigaciones filosóficas sobre la esencia de la libertad humana, más conocido como Freiheitsschrift, Schelling asume la perspectiva, propia de su metafísica intermedia (1804-1820), de un equilibrio relativo entre las fuerzas de contracción y expansión en el mundo ${ }^{1}$. Su formulación de la base que sostiene la realidad en términos de un resto que nunca desaparece, ni logra resolverse en entendimiento nos conducirá a pensar que, para el

\footnotetext{
1 Nuestras citas del Freiheitsschrift corresponden a Friedrich Wilhelm Joseph Schelling, Philosophische Untersuchungen über das Wesen der menschlichen Freiheit und die damit zusammenhängenden Gegenstände (1809), en Schellings Sämtliche Werke (= SW), XIV vols., ed. Karl Friedrich August Schelling (Stuttgart: Cotta, 1856-1861), VII, 331-416. Tras punto y coma indicaremos también la paginación de traducciones castellanas consultadas: Sobre la esencia de la libertad humana y los temas con ella relacionados, trad. Arturo Altman (Buenos Aires: Juárez, 1969). También consultada: Investigaciones filosóficas sobre la esencia de la libertad humana y los objetos con ella relacionados, trad. Helena Cortés y Arturo Leyte (Barcelona: Anthropos, 2000). Todas las traducciones son propias.
} 


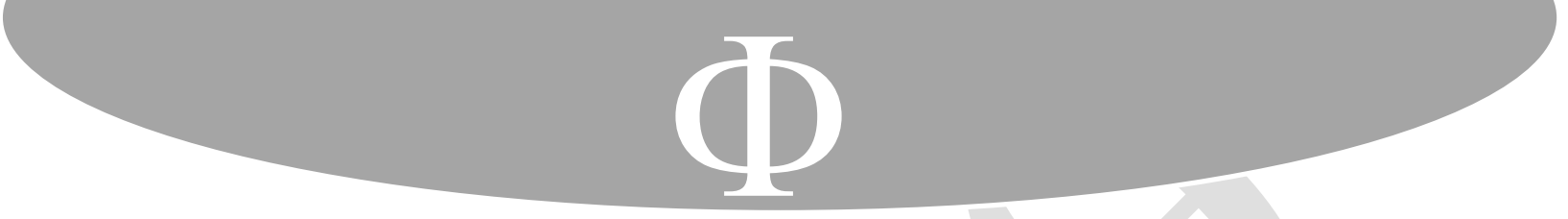

Naturphilosophie una oposición previa, legada por Descartes y Kant, entre res cogitans y res extensa, razón y entendimiento, libertad y naturaleza 5 .

En la medida en que el hombre y la libertad humana constituyen el punto de partida de la reflexión schellinguiana, y, consecuentemente, ésta transita el camino desde lo finito a lo infinito, o, similarmente, de la libertad humana al sistema, desde los entes al mundo, su filosofía profundiza la senda iniciada por el idealismo kantiano, es decir, es una filosofía de la razón finita ${ }^{6}$.

En este trabajo asumimos el concepto de libertad en el sentido más propio y original de Schelling, a saber como «facultad del bien y del mal» (Vermögen des Guten und des Bösen $)^{7}$. Este constituye una amalgama, según Andrew Bowie, de dos teorías. Por un lado, el concepto de libertad como espontaneidad y actividad del idealismo, y, por el otro, una concepción de la naturaleza como vida, devenir y productividad, propio de la Naturphilosophie ${ }^{8}$.

\footnotetext{
${ }^{5}$ Cf. Andrew Bowie, Schelling and modern european philosophy. An introduction (Londres: Routledge, 1993), 94; Ashley Underwood Vaught, The Specter of Spinoza in Schelling's Freiheitsschrift (ProQuest: UMI, 2011), 225 s. A través de una reflexión general sobre la vinculación entre necesidad y contingencia, Miklos Vetö afirma que «Par cette réduction de la nécessité en contingence la Spätphilosophie schellingienne engage le procès de l'idéalisme absolu qu'elle instruira dans l'esprit de Kant, auquel elle renouvelle d'ailleurs son allégeance. Le kantisme est essentiellement la doctrine de la finitude de la Raison, de même que de la primauté du pratique vis-à-vis du théorique. Schelling retrouve ces deux ressorts fondamentaux de la Critique et il les synthétise dans ses investigations. L'essence contingente de la nécessité rationnelle signifie l'échec de prétentions de la Raison Absolue». Miklos Vetö, De Kant a Schelling. Les deux voies de l'idéalisme allemand, 2 vols. (Grenoble: Jérôme Millon, 2000), II: 363.

${ }^{6}$ Este punto es señalado por Lauth al criticar, aunque desde un punto de vista fichteano, al sistema anterior de Schelling, de la identidad, con rasgos marcadamente monista-inmanentes, y panteístas, que su concepción intermedia deja más relegados. Cf. Reinhardt, Lauth, La concepción del sistema de la filosofía en Descartes, trad. Alberto Ciria (Málaga: Servicio de Publicaciones de la Universidad de Málaga y Grupo de Investigación sobre el Idealismo alemán, 2006), 230. También Andrew Bowie, Schelling and modern european philosophy. An introduction., 86; Fernando Pérez-Borbujo Álvarez, Schelling. El sistema de la libertad (Barcelona: Herder, 2004), 16, 26; Ashley Underwood Vaught, The Specter of Spinoza in Schelling's Freiheitsschrift, 5 ss., 156 s., 200 s., en particular 201: «we might also say that human being is transcendent in the traditional sense in relation to created nature, and even that human being transcends this becoming God. That is, if God is dependent upon the destiny of creation (...) then human being becomes the eminent finite existence. (...) finitude no longer denotes what is ontologically secondary, but strangely, what is ontologically primary».

${ }^{7}$ Schellings Sämtliche Werke VII, 352; 94/151.

${ }^{8} \mathrm{Cf}$. Andrew Bowie, Schelling and modern european philosophy. An introduction, 94; Naomi Fisher, «Freedom as Productivity in Schelling's Philosophy of Nature», en Schelling's Philosophy: Freedom, Nature, and Systematicity, ed. G. Anthony Bruno (Oxford: Oxford University Press, 2020), 56-58, 64 ss. Más sobre la relación entre naturaleza, libertad y sistema, que aquí trabajamos tangencialmente, en Andrew Bowie, «Nature and Freedom in Schelling and Adorno», en Interpreting Schelling: Critical Essays, ed. Lara Ostaric (Cambridge: Cambridge University Press, 2014), 180-182, 184 ss., 193; Michelle Kosch, «Idealism and freedom in Schelling's Freiheitsschrift» en Interpreting Schelling: Critical Essays, 145-159; Lara Ostaric, «The Concept of Life in Early Schelling», en Interpreting Schelling: Critical Essays, 48-70.
} 


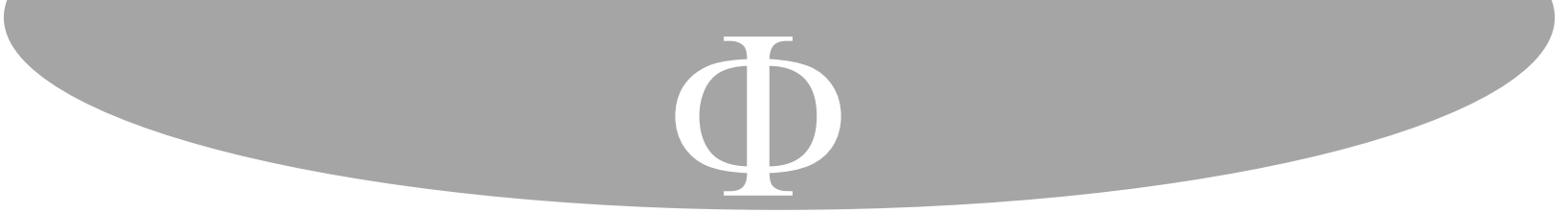

El nuevo curso que emprende la reflexión schellinguiana sobre la libertad buscará esclarecer en el contexto antedicho «cómo se produce en el hombre individual (einzelnen Menschen) la decisión (Entscheidung) para el mal o el bien (...)»9.

Schelling sostiene que la facultad de querer, en un sentido más originario, es contraria a una noción de libertad orientada exclusivamente al bien, pues sin alternativas reales no podemos hablar de una auténtica elección $(W a ̈ h l)^{10}$. El bien como el mal, la posibilidad abierta y no previamente decidida por uno de ellos, son fundamentales para que exista verdaderamente libertad $^{11}$, pues esta requiere que la opción por el bien o el mal permanezca siempre indecidida (unentscheidete) y recaiga, de este modo, sobre la entera disposición metafísico-ontológica y moral del hombre.

A pesar de afirmar que el dualismo no puede ser el punto de vista más elevado de la filosofía, porque impide que esta se erija en sistema, Schelling se esfuerza por articular un tipo de dualidad admisible que dé cuenta, precisamente, de la existencia del mal. Por este motivo, introduce las nociones de «fundamento» y «existencia» (Grund, Existenz). Las mismas constituyen, al decir de Richard Bernstein, «distinciones primarias y esenciales en el Ser» ${ }^{12}$.

Heidegger, nuevamente, nos orienta en la intelección de estos conceptos. Ser mienta la categoría más general, que da origen a la dualidad, pero que no puede mostrase o evidenciarse en cuanto tal ${ }^{13}$. La dualidad se expresa en dos elementos o componentes metafísicos indisolubles pero distinguibles. Por un lado, el concepto de Grund hace referencia a lo no-racional, base o sustrato de todo ente y de toda la realidad. Por el otro, Existenz se predica de los entes bajo cierto aspecto, esto es como presencia objetiva de aquello que se revela ${ }^{14}$.

\footnotetext{
${ }^{9}$ Schellings Sämtliche Werke VII, 382; 136/221.

${ }^{10}$ Cf. Miklos Vetö, De Kant a Schelling. Les deux voies de l'idéalisme allemand, II: 392.

${ }^{11}$ Cf. Martin Heidegger, Schelling: Vom Wesen der menschlichen Freiheit (1809), en Idem., Gesamtausgabe, II. Abteilung: Vorlesungen 1919-1944, Band 42 (Frankfurt am Main: Vittorio Klostermann, 1988). Reproduce las lecciones impartidas en Friburgo sobre el Freiheitsschrift de Schelling del semestre de verano de 1936: Schellings Abhandlung über das Wesen der menschlichen Freiheit (1809), ed. I. Schüssler (Tubinga: Max Niemeyer, 1971). Trad. cast.: El tratado de Schelling sobre la esencia de la libertad humana (1809), trad. M. Dimópulos (Buenos Aires: Waldhuter, 2015), 165. A continuación citamos siempre según la Gesamtausgabe seguida de la paginación castellana tras el punto y coma (= GA 42, 167 s.; 165). Cf. Mariano Gaudio, «Schelling. En el vientre de Dios», en Tras los pasos del mal. Una indagación en la filosofía moderna, comp. Beatriz von Bilderling (Buenos Aires: Eudeba, 2008), 233.

${ }^{12}$ Cf. Richard Jacob Bernstein, El mal radical. Una indagación filosófica, trad. Marcelo G. Burello (Buenos Aires: Lilmod, 2005), 123.

${ }^{13}$ Sobre el rol de la exégesis de Heidegger de la filosofía intermedia de Schelling cf. Emilio Carlo Corriero, The Absolute and the Event. Schelling after Heidegger, trad. Vanessa Di Stefano (Londres/ Nueva York: Bloomsbury, 2020), 27-80; Sylvaine Gourdain, «Das Ethos des Denkens, ein Ethos der Muße. Überlegungen im Anschluss an Heidegger und Schelling», en Anthropologie der Theorie, eds. Thomas Jürgasch y Tobias Keiling (Tübingen: Mohr Siebeck, 2017), 275-295.

${ }^{14}$ Cf. Schellings Sämtliche Werke VII, 357; 101/161, 163; GA 42, 186-188; 182 s.; Miklos Vetö, De Kant a Schelling. Les deux voies de l'idéalisme allemand, II: 312 ss. Véase también Markus Gabriel, «Schelling on the
} 


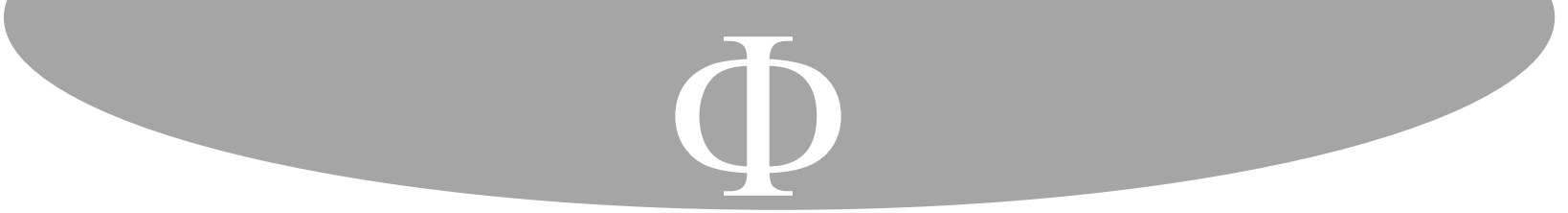

La dualidad interna de Grund y Existenz se erige a partir de este punto en un doble principio de todos los seres, es decir, de Dios, del hombre y de la naturaleza ${ }^{15}$. Con la distinción aplicada especialmente a Dios nuestro autor emprende la fundamentación de su diferencia ontológica con el mundo, lo que a su vez le permite afirmar la independencia de lo finito, la movilidad de la naturaleza y, finalmente, la existencia de la libertad humana.

La intención de nuestro autor al introducir los conceptos de absolutidad derivada y dependencia autónoma para dar cuenta de la libertad finita, así como luego los de fundamento y existencia, es preservar para la libertad, como para toda la naturaleza, una raíz independiente de Dios que posibilita, precisamente, su autosubsistencia (Selbständigkeit) ${ }^{16}$.

El de Leonberg se vale de un doble concepto inmanente y trascendente de libertad humana que es necesario esclarecer para comprender el desarrollo posterior del trabajo.

La libertad, como todo lo finito, incluyendo a la naturaleza, resulta inmanente, en la medida en que se halla en el devenir del fundamento, el cual posee una raíz independiente de Dios. Es trascendente, sin embargo, en la medida en que opera «fuera» (Extra) o «más allá» (Praeter) del fundamento de la naturaleza y es libre respecto de este. Dios, por su parte, es también trascendente a lo finito, como veremos, porque no se encuentra en relación de equivalencia con la naturaleza, como el Deus sive natura spinoziano, sino en una diferencia ontológica radical o toto genere ${ }^{17}$.

Es menester detenernos un poco más en los múltiples usos de que nuestro autor se vale cuando utiliza los conceptos de inmanencia y trascendencia. Estas aclaraciones son importantes para valorar la principal aportación metafísica del Freiheitschrift, esta es, la formulación de un concepto diferenciado de inmanencia que posibilita la existencia por sí de lo finito en general, así como del mundo y del hombre en particular.

Compatibility of Freedom and Systematicity», en Schelling's Philosophy: Freedom, Nature, and Systematicity, 137 s. quien señala acertadamente la centralidad de la cuestión de la esencia (Wesen) como polo de unidad ínsito en la dualidad misma de fundamento y existencia, así como en la pregunta por la libertad humana (Wesen der menschlichen Freiheit).

${ }^{15}$ Cf. Schellings Sämtliche Werke VII, 362 ss.; 108 ss./175 ss.; Friedrich Hermanni, Die letzte Entlassung. Vollendung und Scheitern des abendländischen Theodizee-proyektes in Schellings Philosophie (Viena: Passenger Verlag, 1994), 85-113.

${ }^{16}$ Cf. Michel Theunissen, «Schellings anthropologischer Ansatz», Archiv für Geschichte der Philosophie, 47 (1965): 181 s. Tomamos la sugerencia de traducir el alemán Selbständigkeit como «autosubsistencia» en vez de como «autonomía» (Autonomie) de los comentarios del Dr. Alberto Damiani.

17 Tampoco servirá aquí atenerse al punto de vista de la filosofía de la identidad que busca reformular el Deus sive natura spinoziano por el postulado idealista-racionalista absoluto de Deus sive Vernunft. De lo que se trata a partir de la nueva perspectiva schellinguiana abierta en 1804/1809 es de concebir la tragedia, por así decir, del Deus sive nihil que deja al hombre y a su libertad en una radical independencia que es también una situación de orfandad respecto del principio. Cf. Stuttgarter Privatvorlesungen, en SW VII, 429. Sobre el primer punto véase Yitzhak Y. Melamed, «Deus Sive Vernunft. Schelling's Transformation of Spinoza's God», en Schelling's Philosophy: Freedom, Nature, and Systematicity, 93 s., 103-107. 


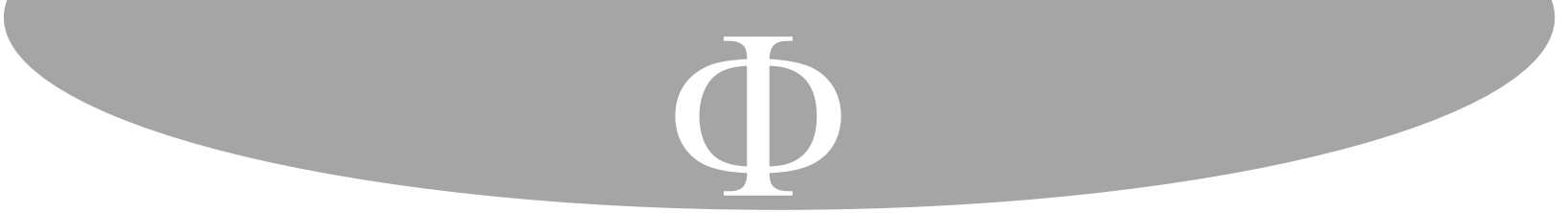

abre un hiato inexplicable entre, por así decir, el Dios que es y el Dios que deviene, o heideggerianamente, entre Dios y el hombre ${ }^{22}$.

El Freiheitsschrift evidencia en la discusión sobre el panteísmo y el problema del acosmismo, la dificultad que la filosofía moderna encuentra a la hora de hacer justicia a las prerrogativas tanto de lo finito como de lo infinito. El planteo del nuevo sistema de Schelling busca pensar ambos polos sin reducirlos unilateralmente a unidad. Por un lado, pone de manifiesto la imposibilidad última de introducir diferencias en una filosofía radicalmente monista-inmanente. Al mismo tiempo se atiene a la doctrina idealista de que la razón humana debe reconocer la independencia y autosubsistencia de lo finito en general $^{23}$.

En las primeras líneas del escrito sobre la libertad Schelling asume un punto de vista metafísico que se convierte, gradualmente y con el desarrollo de la argumentación, en otro de índole moral y antropológica. A continuación, nos concentraremos en este segundo aspecto de la investigación schellinguiana.

Nuestro autor aboga por una forma de realización del bien distinta de la equivalencia entre realidad y perfección. El proceso de separación de la realidad del en-sí de Dios constituye el fundamento por el cual el bien, ubicado fuera y más allá de la idealidad original del principio, llega a ser por sí superando al estado de indiferencia inicial ${ }^{24}$.

Bajo el prisma de la nueva posición schellinguiana la existencia del mundo será el resultado de una decisión de Dios por el bien que es consumable sólo en y por el hombre. De esta manera encuentran resolución el problema ontológico de la distinción y la unidad, por

Spinoza in Schelling's Freiheitsschrift, 188, 203 ss. Respecto del concepto de «diferencia absoluta» en relación con la incompletitud de la identidad lógica, cf. Luis Fernando Cardona, «Acto inteligible y realidad individual del "malum morale"», 512 ss.

${ }^{22}$ Cf. GA 42, 190 s., 195 s., et. al.; 186 s., 191 s., et. al.; Andrew Bowie, Schelling and modern european philosophy. An introduction, 92; Arturo Leyte y Völker Rühle, «Estudio introductorio», en Friedrich Wilhelm Joseph Schelling, Investigaciones filosóficas sobre la esencia de la libertad humana, 54 ss. Ana Carrasco Conde, La limpidez del mal, 253. Es importante destacar que la crítica posterior de Schelling a Spinoza y Hegel, pero en general a todo sistema monista-inmanente, tiene que ver con la imposibilidad de estos de dar cuenta del devenir real, es decir, de un movimiento que produce más de lo que está contenido en el mero desenvolvimiento lógico del concepto y que nos induciría a considerar la realidad como un proceso ya acabado o cerrado, cancelando la libertad humana y la movilidad de la naturaleza y la historia. Cf. GnP, en Schellings Sämtliche Werke X, 124 s.; 217 s.; Ignacio Falgueras Salinas, «Estudio introductorio», en Friedrich Wilhelm Joseph Schelling, Lecciones muniquesas para la historia de la filosofía moderna, 54; Markus Gabriel, Transcendental Ontology: Studies in German Idealism (London: Continuum, 2011), 61 ss., 82-96.

${ }^{23} \mathrm{Cf}$. El análisis de la imposibilidad de un tránsito de lo infinito a lo finito en Spinoza que ofrece Schelling en sus lecciones muniquesas, en Schellings Sämtliche Werke X, 41 ss.; Richard Jacob Bernstein, El mal radical. Una indagación filosófica, 120; Miklos Vetö, De Kant a Schelling. Les deux voies de l'idéalisme allemand, II: 475.

${ }^{24}$ Cf. Miklos Vetö, De Kant a Schelling. Les deux voies de l'idéalisme allemand, II: 393. También en II: 396 : «Les Recherches [se refiere al Freiheitsschrift] écrivent que le formel provient du positif. C'est dire que la positivité du mal ne se rapporte pas à un contenu fabriqué para la liberté humaine, mais à une nouvelle organisation $[\ldots]$ du donné»». 


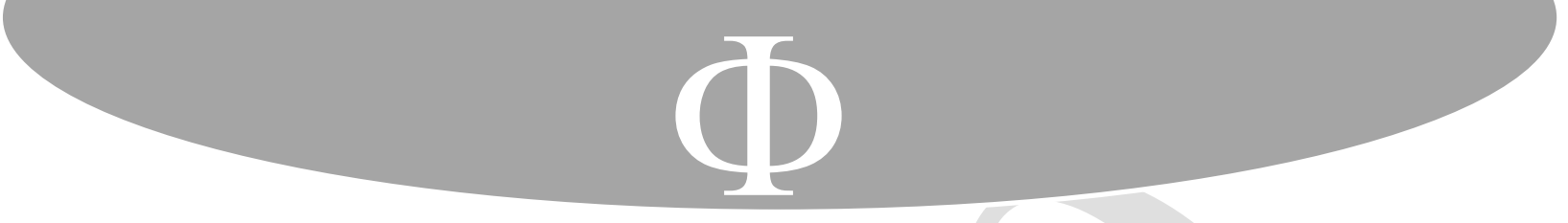

pues, no es posible una superación definitiva de la antítesis entre bien y mal que lo sitúe más allá (Praeter) de la dualidad, esto es, de la realidad (Realität $)^{40}$.

La distinción principal en este punto del Freiheitsschrift comienza a recaer sobre el concepto de espíritu (Geist). Este introduce la idea de una voluntad por encima y por fuera de toda naturaleza. Schelling denomina a esta voluntad «personalidad» (Persönlichkeit), y es posible en el hombre porque en su seno la mismidad o particularidad es elevada a lo ideal ${ }^{41}$. Esta particularidad o mismidad ideal es también caracterizada como una voluntad que actúa con plena libertad (völligen Freiheit) y, por lo tanto, se sustrae de la voluntad universal que impera en la naturaleza (Universalwillens). Así como lo real y efectivo característico de lo finito se situaba fuera y más allá del principio ideal ahora Schelling coloca al hombre por encima y por fuera (über und außer) de toda naturaleza y voluntad universal ${ }^{42}$.

Los conceptos de personalidad y particularidad son de suma importancia en el decurso subsiguiente del Freiheitsschrift porque permiten al de Leonberg articular una noción fuerte de autosubsistencia del hombre frente al orden natural, por un lado, y respecto de Dios, por el otro ${ }^{43}$. Esta subsistencia por sí del hombre respecto de Dios es otra forma de aludir al fenómeno que hemos denominado su destinación, respecto de la cual nos ocuparemos a continuación, en el siguiente apartado de este trabajo.

${ }^{40}$ Cf. Schellings Sämtliche Werke VII, 364; 111/179; Mariano Gaudio, «Schelling. En el vientre de Dios», 246 s. Tampoco, en consecuencia, el hombre podrá superar su libertad propia, y, en este sentido, su responsabilidad moral. Esta inexorabilidad de la libertad humana es descrita por Heidegger como una relación interna y esencial entre posibilidad y finitud: „Wählen heisst auf Möglichkeiten sich beziehen und dabei die eine der anderen vorziehen. Wählen können bedeutet daher endlich sein müssen. (...) Die Vollkommenheit des Absoluten besteht im Gegenteil darin, nur eines wollen zu können, und diese Eine ist die Notwendigkeit seines eigensten Wesens. Und dieses Wesen ist die Liebe." GA 42, 276 s.; 266. En similar tono, cf. Slavoj Zizek, The indivisible remainder. An essay on Schelling and related matters, 60. Según el autor esloveno, la libertad se relaciona con la incompletitud del hombre, en el ámbito antropológico y moral, y con la indecisión y la dualidad, en sentido metafísico. El ser humano se halla en la disyuntiva entre cursar una proto-existencia, meramente ideal-nocional, o bien existir realmente, pero como «otro». Aquí Zizek vincula la libertad con la alienación en sentido práctico y psicológico. Más aún, la inversión del hombre se constata, para este autor, en que todo lo metafísicamente elevado le aparece como «espectral», y esto porque no es solamente, como dijimos, un ente finito-infinito, sino, más bien, ni finito ni infinito.

${ }^{41}$ Si bien Schelling trabaja el concepto de espíritu (Geist) por lo menos desde el System des transzendentalen Idealismus de 1800, no es el caso de la noción de personalidad (Persönlichkeit) a la que rechazaba desde una perspectiva spinozista contraria a la libertad y la individualidad y afín al concepto de sistema como totalidad inmanente que pone la diferencia como inesencial. Cf. Vom Ich, en Schellings Sämtliche Werke I, 200; Schellings Sämtliche Werke VII, 333 s., 412.

${ }^{42}$ Cf. Schellings Sämtliche Werke VII, 364; 111/179. Véase también Slavok Zizek, The indivisible remainder. An essay on Schelling and related matters, 57-59, 64.

${ }^{43}$ Sobre el concepto de personalidad en Schelling cf. Ana Carrasco Conde, La limpidez del mal, 115, 117. La definición de personalidad (Persönlichkeit) en la obra de 1809 se encuentra en Schellings Sämtliche Werke VII, 364; 111/179: „Die Selbstheit als solche ist Geist, oder der Mensch ist Geist als ein selbstisches, besonderes (von Gott geschiedenes) Wesen, welche Verbindung eben die Persönlichkeit ausmacht.“(Énfasis original). 


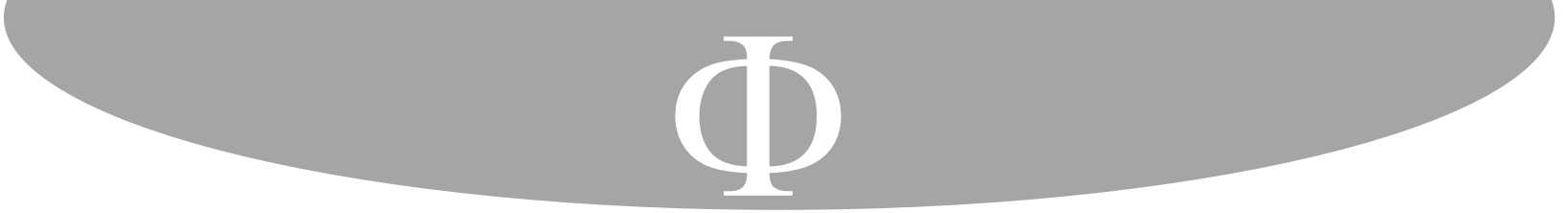

Anteriormente, Schelling se había manifestado sobre el mismo fenómeno de tensión inherente que sostiene a la naturaleza a partir de las nociones de gravedad y de luz. La gravedad, como el fundamento, constituye un eterno fondo oscuro anterior a la luz en tanto principio de lo existente y nunca puede ser por completo esclarecida por ésta ${ }^{51}$.

En el ámbito ideal, la tensión adopta la forma de una dialéctica entre las voluntades de amor y de fundamento. Ambas constituyen, según nuestro autor, las dos caras de la voluntad de revelación de Dios (Wille der Offenbarung), o, lo que es lo mismo, de la existencia de lo finito entendido como realidad extra et praeter Deum. La tensión entre fundamento y revelación es también una dialéctica que sólo puede avanzar exponiéndose contra su contrario, por ejemplo, el amor y la revelación, que constituyen formas de lo universal, sólo se tornan patentes en la particularidad que conduce al mal. La separación de ambos principios no excluye su unión, que, paradójicamente, únicamente puede ser tal porque cada uno se esfuerza por actuar para si ${ }^{52}$.

Resulta pertinente considerar aquí la relación entre Dios y el bien y el mal que se manifiestan a partir del hombre y de la voluntad de revelación que éste debe llevar a su realización.

Es menester dilucidar, asimismo, cómo se compatibiliza esta última posición con la doctrina, defendida por nuestro autor en el Freiheitsschrift, de la independencia del mal y del fundamento respecto de Dios como existencia absoluta. ¿En qué sentido podemos decir esto? ¿De qué modo se siguen el bien - y el mal - del fundamento?

Debemos puntualizar que no es posible deducir linealmente el bien de Dios y el mal del fundamento: «tampoco puede afirmarse que el mal provenga del fundamento, o que la voluntad del fundamento sea autor de aquél, puesto que el mal sólo logra surgir en la más íntima voluntad del corazón y nunca se consuma sin un acto propio (eigne That)» ${ }^{53}$. El

der verbesserten Fichteschen Lehre (1806), en Schellings Sämtliche Werke VII, 54. Sobre la dependencia del concepto de razón del de Grund, cf. Miklos Vetö, De Kant a Schelling. Les deux voies de l'idéalisme allemand, II: 348 .

${ }^{51}$ Cf. Schellings Sämtliche Werke VII, 358; 102/163. Cf. Schellings Sämtliche Werke III, 31, 43, 298, IV, 181, VI, 320. Como bien afirma Miklos Vetö, De Kant a Schelling. Les deux voies de l'idéalisme allemand, II: 318): «La nature en tant que processus de production est matrice de produits mais elle ne concède à ses rejetons qu'une existence précaire et provisoire. (...) est une activité fiévreuse mais qui ne cherche qu'à retourner au repos de l'identité. C'est un principe de la particularité et de la différence mais que dans tous ses efforts ne vise que la dissolution dans la fluidité, le retour à l'indifférence. Productivité opposée au produit (...) l'ennemi implacable de l'existence séparée. Elle s'oppose à l'individualité, elle combat surtout la figure articulée».

52 Cf. Schellings Sämtliche Werke VII, 375; 126 s./205, 207. Heidegger expresa también la interdependencia entre, en su caso, fundamento y revelación, cerrazón sobre sí y escisión de fuerzas. Cf. GA 42, 231-238; 225230. Asimismo Ana Carrasco Conde, La limpidez del mal, 76 ss., 94, 131, 235, 269.

53 Schellings Sämtliche Werke VII, 399; 161/263, 265: „es kann auch nicht gesagt werden, daß das Böse aus dem Grunde komme, oder daß der Wille des Grundes Urheber desselben sey. Denn das Böse kann immer nur entstehen im innersten Willen des eignen Herzens, und wird nie ohne eigne That vollbracht." Cf. Luis Fernando Cardona, «Acto inteligible y realidad individual del "malum morale"», 513-520. 


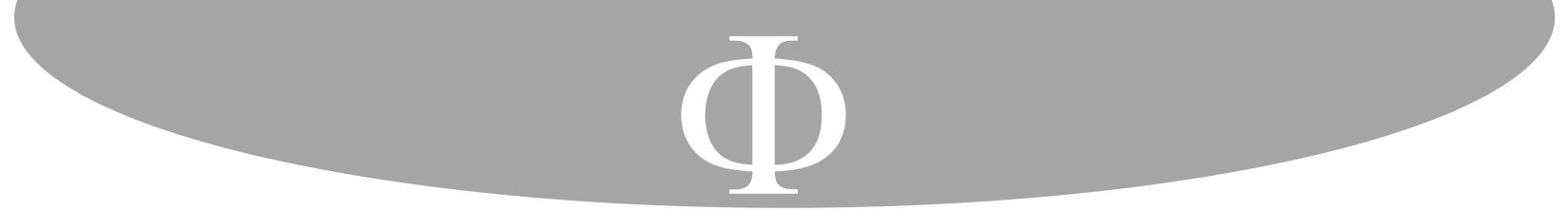

En donde todos los opuestos no están tanto unificados (vereinigt) como uno (eins), ni son superados (aufgehoben) en cuanto tales, sino que no están separados en absoluto (gar nicht getrennt sind). (...) allí donde unidad y oposición, lo idéntico consigo mismo y su contrario son uno ${ }^{58}$.

En consecuencia, tanto el bien como el mal son formas de unidad que presuponen la distinción y separación entre existencia y fundamento y, concretamente, entre los principios de amor y mismidad. El bien y el mal «derivan» del fundamento no según una deducción lineal, sino por cuanto es a partir suyo que se despliega una diferenciación en el Dios de la identidad absoluta bajo las formas de la oscuridad y la luz, mismidad y amor, de cuya interrelación surgen finalmente aquellos. Schelling había expresado estas ideas ya en la parte cosmogónica del escrito de 1809. Allí nos decía que el anhelo que corresponde al fondo oscuro es la primera forma de existencia divina. Ésta genera una representación reflexiva interna (innere reflexive Vorstellung) gracias a la cual el principio de entendimiento o luz produce la originaria separación de las fuerzas (Scheidung der Kräfte) ${ }^{59}$.

Villacañas nos habla, en su obra sobre el idealismo alemán, de la contraposición entre las nociones de voluntad en Schelling y Schopenhauer. Si bien ambos autores conceden a este concepto una centralidad fuerte en sus filosofías en tanto principio ciego y expansivo, Schelling no cesa jamás de vincular la voluntad o el principio oscuro con el entendimiento. Se trata de una voluntad que busca y anhela el entendimiento, como en la historia buscamos un bien desconocido y anónimo, a diferencia de Schopenhauer para quien esta noción constituye, más bien, una fuerza ciega e indómita ${ }^{60}$.

Con su caracterización del absoluto y de su salida de sí a partir de la dualidad, Schelling nos conduce más allá de la posibilidad de la libertad humana y el mal hacia dominios del ser determinados, como la naturaleza y la historia, ambas facetas de la creación.

\footnotetext{
${ }^{58}$ Schellings Sämtliche Werke IV, 235 s.: ,worin alle Gegensätze, nicht sowohl vereinigt, als vielmehr eins, und nicht sowohl aufgehoben, als vielmehr gar nicht getrennt sind? (...) worin die Einheit und der Gegensatz, das sich selbst Gleiche mit dem Ungleichen eins ist.“ Cf. Ana Carrasco Conde, La limpidez del mal, 66-74. Para la transformación de lo Uno en dualidad por intermedio de la voluntad de amor (Wille der Liebe), cf. Schellings Sämtliche Werke VII, 406-408; 171-174/279-285; GA 42, 280 s.; 270; Fernando Pérez-Borbujo Álvarez, Schelling. El sistema de la libertad, 58-61.

${ }^{59}$ Cf. Schellings Sämtliche Werke VII, 360 s.; 105 s./171. Cuando el ser primigenio se niega, deviene él mismo objeto de su deseo y se escinde en dos esencias. Cf. Die Weltalter, en Schellings Sämtliche Werke VIII, 223 s.; GnP, en Schellings Sämtliche Werke X, 101; 198: ,aber es ist ihm unvermeidlich, sich sich selbst anzuziehen, denn nur dazu ist es Subjekt, daß es sich selbst Objekt werde, da vorausgesetzt wird, daß nichts außer ihm sey, das ihm Objekt werden könne; indem es sich aber sich selbst anzieht, ist es nicht mehr als nichts, sondern als Etwas - in dieser Selbstanziehung macht es sich zu etwas; in der Selbstanziehung also liegt der Ursprung des Etwas-Seyns, oder des objektiven, des gegenständlichen Seyns überhaupt. Aber als das, was es Ist, kann sich das Subjekt nie habhaft werden, denn eben im sich-Anziehen wird es ein anderes, dieß ist der GrundWiderspruch [...]“ (Énfasis original).

${ }^{60}$ Cf. José Luis Villacañas, La filosofía del idealismo alemán, 2 vols. (Madrid: Síntesis, 2001), II: 186 s. La confirmación de esta posición referida a la derivación indirecta del bien y el mal del fundamento y, por lo tanto, del ser-en Dios, en Schellings Sämtliche Werke VII, 409; 175/287.
} 


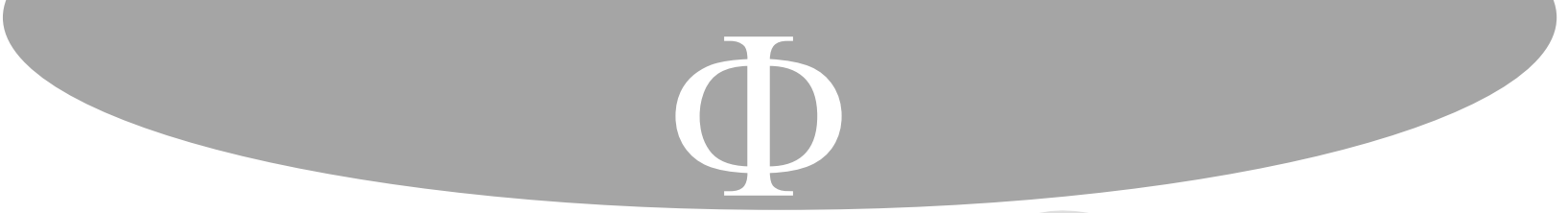

Consideremos pues el desarrollo subsiguiente como una exégesis del siguiente fragmento de la obra de 1809:

Eso acontece [la oposición del espíritu del amor al espíritu del mal] sólo mediante la revelación (Offenbarung), en el sentido más estricto de la palabra, que necesita tener las mismas fases que la primera manifestación en la naturaleza, de suerte que también en este caso la cumbre más alta de la revelación es el hombre, más el hombre arquetipo y divino (urbildliche und göttliche Mensch), aquel que al principio estaba junto a Dios, y en quien se crearon todas las cosas y el hombre mismo ${ }^{61}$.

\section{Finitud, mal y pérdida de la universalidad}

En los pasajes analizados, el autor del Freiheitsschrift ha dilucidado la interrelación dialéctica entre amor y fundamento o mismidad, en Dios, el hombre y la creación original. En el decurso posterior de la naturaleza y la historia, inclinarán el desarrollo hacia la relativa armonía de ambos - como en el bien- o en dirección a cierto desbalance o descentramiento como en el mal- ${ }^{62}$.

En la naturaleza, hallamos indicios de la voluntad de fundamento en los elementos irracionales y contingentes que intervienen en la creación de la criatura. Estos indican siempre, al decir del de Leonberg, la acción de una voluntad particular y libre ${ }^{63}$.

61 Schellings Sämtliche Werke VII; 377; 129 s./211: „Dieß geschieht allein durch die Offenbarung, im bestimmtesten Sinne des Worts, welche die nämlichen Stufen haben muß wie die erste Manifestation in der Natur, so nämlich, daß auch hier der höchste Gipfel der Offenbarung, der Mensch, aber der urbildliche und göttliche Mensch ist, derjenige, der im Anfang bei Gott war, und in dem alle anderen Dinge und der Mensch selbst geschaffen sind." Aquí encontramos la doctrina de la caída del hombre y del mundo, esto es, una reflexión sobre de qué modo la decisión del hombre, que implica una caída, arrastra consigo a toda la naturaleza. Fackenheim nos explica que sólo el hombre, al comienzo de su existencia y durante ella, se ubica en la cornisa de la decisión. Esta se manifiesta como la posibilidad original entre actuar o no actuar. En efecto, para querer algo primeramente es necesario determinarse a querer algo en general: si bien empíricamente no podemos representarnos un «querer-querer», o un querer nuestro deseo, la situación de indecisión originaria del hombre se manifiesta del modo antedicho. Al decidirse a actuar, el hombre introduce un cambio en la creación de Dios entendida como naturaleza, y esta modificación arrastra a toda la creación en la medida en que sólo a través del hombre Dios se vincula a aquella y puede reconocerla como semejante a Él, como personalidad y espíritu. Emil Ludwig Fackenheim, «Schelling's Conception of Positive Philosophy», 573 s., 576, 579; Sylvaine Gourdain, «Au-delà de la nécessité et de la contingence: la liberté absolue dans la philosophie tardive de schelling, "liberté d'être et de ne pas être"», Les Études philosophiques, No. 4, Actualité de la théologie politique? (Presses Universitaires de France, Octobre 2014): 573-588. Respecto de la imposibilidad de «querer querer» o querer el propio deseo, cf. Arthur Schopenhauer, Los dos problemas fundamentales de la ética, trad. Pilar López de Santa María (Madrid: Siglo XXI, 2002), 49

${ }^{62}$ Cf. Schellings Sämtliche Werke VII, 370 s.; 119 s./193, 195; Slavoj Zizek, The indivisible remainder. An essay on Schelling and related matters, 63.

${ }^{63}$ Cf. Schellings Sämtliche Werke VII, 376; 127/207; Ashley Underwood Vaught, The Specter of Spinoza in Schelling's Freiheitsschrift, 267, 275. 


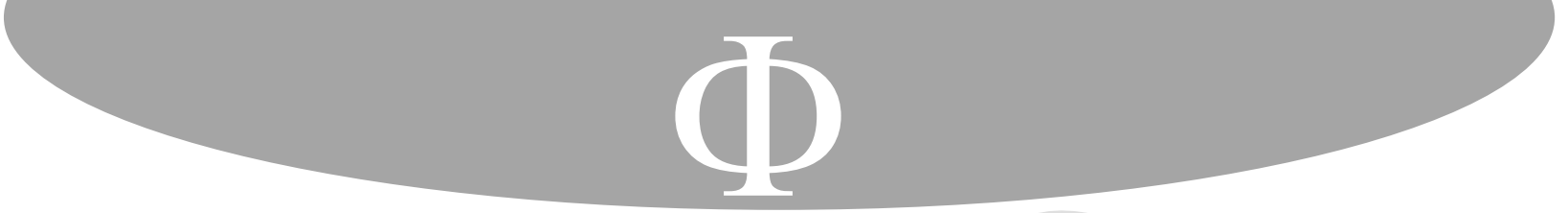

Todo impulso natural y deseo, aspira a la libertad al tiempo que se origina en ella. En palabras de Schelling:

Aun el concepto de base (Basis) (...) tiene que conducir, científicamente considerado, al concepto de mismidad (Selbstheit) y yoidad (Ichheit). Pero en la naturaleza hay determinaciones contingentes (zufällige Bestimmungen) que sólo cabe explicar a base de una conmoción del principio irracional (irrationalen) u oscuro (finstern) (...) por la mismidad que se ha tornado activa ${ }^{64}$.

Para nuestro autor, el mal se anuncia en la creación por su efecto, esto es, a causa del hombre, y la característica del espíritu reside en que los principios de luz y oscuridad, amor y mismidad, se articulan en él de modo personal. El bien, por lo tanto, se origina también en el espíritu bajo figura personal y humana. El hombre es, en efecto, esta figura que cumple un rol de mediador entre la naturaleza y Dios, escindidos tras la creación, para lograr la autosubsistencia de lo finito. La mediación asume la forma de un restablecimiento de la personalidad y espiritualidad de Dios mismo, que se revela en la naturaleza, gracias a la personalidad y espiritualidad humanas ${ }^{65}$.

Podemos decir que el principio del mal, según Schelling, tiene su origen metafísico en la dureza de toda vida que se orienta a lo universal. Esta tensión conduce al individuo a querer recortarse y salir a la periferia, para autoerigirse en centro. En este re-creado falso

\footnotetext{
${ }^{64}$ Schellings Sämtliche Werke VII, 376; 128/207, 209: „Auch der durch Empirie aufgefundene Begriff der Basis [...] muß, wissenschaftlich gewürdigt, auf den Begriff der Selbstheit und Ichheit führen. Aber es sind in der Natur zufällige Bestimmungen, die nur aus (...) des irrationalen oder finstern Princips der Creatur nur aus aktivirter Selbstheit erklärlich sind.“

${ }^{65}$ Cf. Schellings Sämtliche Werke VII, 380; 134 s./217; Christoph Schulte, «Zimzum in the Works of Schelling», The Jerusalem Philosophical Quarterly. S. H. Bergman Center for Philosophical Studies, 41 (1992): 37; Michel Theunissen, «Schellings anthropologischer Ansatz», 174-176, 186 s. Según este intérprete el punto de vista antropológico es exclusivo del Freiheitsschrift. Anteriormente, Schelling concebía un absoluto impersonal y no-humano y al hombre que aspiraba a él le imponía la destrucción de su personalidad: „Doch streb er damit, wie Schelling betont, nach 'Zernichtung' der Persönlichkeit (Vom Ich, en SW I, 200), also nach Überwindung seines menschlichen Bewusstseins“. Michel Theunissen, «Schellings anthropologischer Ansatz», 175 s. Al decir de Metzger, „Auf den ersten Blick möchte in der Tat in Schellings frühesten Schriften eher die Absolutheit als eine Bestimmung der Ichheit, den das 'Ich' als ein Merkmal des 'Absoluten' erscheinen." Die Epochen der Schellingschen Philosophie von 1795 bis 1802 (Heidelberg: Carl Winter's Universitätsbuchhandlung, 1911), 26. Cf. Mario Gómez Pedrido, «El caso de Schelling en los inicios de su filosofía práctica: la tragicidad de la libertad humana», en El vuelo del búho: estudios sobre filosofía del idealismo, comps. Silvia del Luján di Sanza y Diana Maria López (Buenos Aires: Prometeo, 2014), 337-341; Ashley Underwood Vaught, The Specter of Spinoza in Schelling's Freiheitsschrift, 292. En el Freiheitsschrift hablamos, por el contrario, de una sumisión trágica de Dios al destino humano. En sentido contrario a la interpretación de Theunissen sobre la exclusividad del punto de vista antropológico en 1809, cf. Michelle Kosch, Freedom and Reason in Kant, Schelling, and Kierkegaard, 90 s., 105 ss. Una valoración positiva de la vinculación entre antropomorfismo y libertad en Miklos Vetö, De Kant a Schelling. Les deux voies de l'idéalisme allemand, II: 327.
} 


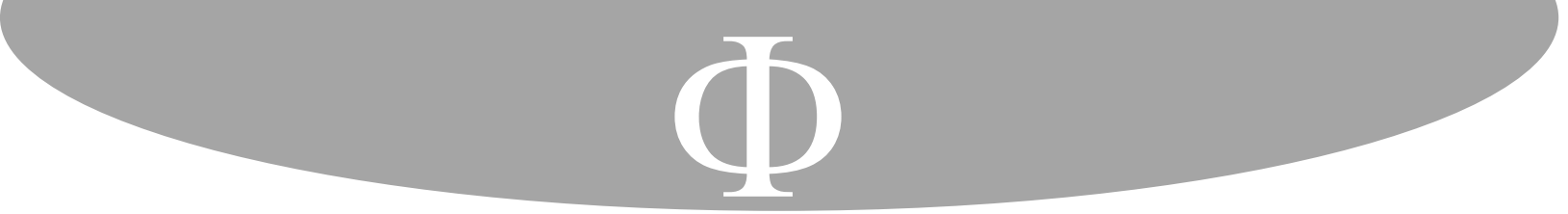

Schelling, Friedrich Wilhelm Joseph. Sobre la esencia de la libertad humana y los temas con ella relacionados. Traducido por Arturo Altman. Buenos Aires: Juárez, 1969.

Schelling, Friedrich Wilhelm Joseph. Investigaciones filosóficas sobre la esencia de la libertad humana y los objetos con ella relacionados (1989). Traducido por Helena Cortés y Arturo Leyte. Barcelona: Anthropos, 2000.

Schelling, Friedrich Wilhelm Joseph. Lecciones muniquesas para la historia de la filosofía moderna. Traducido por Luis de Santiago Guervós. Málaga: Edinford, 1993.

Schopenhauer, Arthur. Los dos problemas fundamentales de la ética, trad. Pilar López de Santa María. Madrid: Siglo XXI, 2002.

Schulte, Christoph. «Zimzum in the Works of Schelling». The Jerusalem Philosophical Quarterly. S. H. Bergman Center for Philosophical Studies 41 (1992): 21-40.

Spielrein, Sabina. «Die Destruktion als Ursache des Werdens». Jahrbuch für psychoanalytische und psychopathologische Forschungen. Editado por Joseph Breuer, Sigmund Freud, Carl Jung 4 (1912): 465-503.

Theunissen, Michel. «Schellings anthropologischer Ansatz». Archiv für Geschichte der Philosophie 47 (1965): 174-189.

Underwood Vaught, Ashley. The Specter of Spinoza in Schelling's Freiheitsschrift. ProQuest: UMI, 2011.

Velkley, Richard. «The Personal, Evil, and the Possibility of Philosophy in Schelling's Freiheitsschrift». En Schelling's Philosophy: Freedom, Nature, and Systematicity. Editado por G. Anthony Bruno, 154-167. Oxford: Oxford University Press, 2020.

Vetö, Miklos. De Kant a Schelling. Les deux voies de l'idéalisme allemand, 2 vols. Grenoble: Jérôme Millon, 2000.

Villacañas, José Luis. La filosofía del idealismo alemán, 2 vols. Madrid: Síntesis, 2001.

Zizek, Slavoj. The indivisible remainder. An essay on Schelling and related matters. Londres: Verso, 2007.

Enviado: 16 de septiembre de 2020 Aceptado: 13 de octubre de 2020 